\title{
Use of Isoproturon Imprinted Polymer Membranes as a Selective Recognition Platform in a Resistance Based Electrochemical Sensor
}

\author{
Krishna Pal Singh ${ }^{1}$, Ramesh Kumar Prajapati ${ }^{1}$, Saurabh Ahlawat ${ }^{2}$, Sweeti Ahlawat ${ }^{1}$, \\ Manjul Mungali ${ }^{1}$, Satish Kumar ${ }^{1}$ \\ ${ }^{1}$ Membrane Biophysics \& Nanobiosensor Research Laboratory, CBSH, G.B. Pant University of Agriculture \& Technology, \\ Pantnagar, India \\ ${ }^{2}$ Clay Product Environment Study Technology Division, CSIR-Central Building Research Institute, Roorkee, India \\ Email: *kps_biophysics@yahoo.co.in,srv.cbri@gmail.com
}

Received December 5, 2012; revised January 10, 2013; accepted January 19, 2013

\begin{abstract}
Methacrylic acid with isoproturon has been utilized to prepare molecularly imprinted polymeric (MIP) membrane on Whatman filter paper no. 5 for selective electrochemical estimation of isoproturon pesticide. MIP membrane was prepared by radical polymerization and characterized using Ultra Violet spectrophotometer, Fourier Transform Infra Red (FTIR) spectroscopy and Scanning Electron Microscopy (SEM). Contact angle study was used to determine the surface energy of the MIP membrane and electrical conductivity measurements were performed by applying a small-amplitude alternating voltage $(20 \mathrm{mV})$ with frequencies varying from $20 \mathrm{~Hz}$ to $80 \mathrm{kHz}$ generated by a low-frequency wave form generator. Different isoproturon concentrations in samples were tested and analyzed. Results indicate linear increase in membrane resistance with increasing isoproturon in $10^{-3}$ to $10^{-6} \mathrm{M}$ range. The selectivity of the electrochemical sensor was confirmed by testing isoproturon in presence of the structurally related compounds monouran and diuran. Results reveal highly selective and sensitive sensor, which can be employed for regular estimation of isoproturon in fields.
\end{abstract}

Keywords: Sensor; Isoproturon; Molecular Imprinting; Monouran; Diuran

\section{Introduction}

Pesticides, such as insecticides, fungicides, and herbicides are widely used in agriculture and horticulture. However they have numerous negative effects like environmental persistence, impact on beneficial organisms and toxicity in humans. Such harmful effects enforce/compel strict management of pesticide use, thus there is a great need for their specific detection and therefore, sensitive, rapid and reliable determination methods are needed. Some standard analytical techniques, such as HPLC, GC- or LC-MS fulfill these requirements up to an extent, and are well-established for many different substances [1-5]. Another approach is based on the high selectivity of antibodies and a number of immunoassays have been developed for the purpose of herbicide determination [6-8]. Despite the excellent sensitivity and selectivity, the uses of such methods are limited because of the relatively long time analysis and the high equipment cost. The apparent attraction of immunosensors is strongly diminished by involved complicated procedure for antibody immobilization; their limited stability and their poor compatibility with transduction

${ }^{*}$ Corresponding author. technology. In order to overcome these limitations, alternative techniques have recently been extensively developed, which include the use of artificial receptors. These artificial receptors mimic the active sites of antibodies.

Molecular imprinting to prepare molecularly imprinted polymers (MIP) has recently gained attention as an appropriate technique in order to generate such receptor active sites in synthetic polymers $[9,10]$. In this approach cross-linked polymers are formed around a template molecule and then template is removed leaving molecular cavities capable of binding the template molecules back. Moreover, the synthesis of MIPs is a straightforward and inexpensive procedure. MIPs demonstrate good thermal and mechanical stability and can be used in aggressive media [11]. The use of MIPs in sensor applications has been demonstrated by different workers [12, 13].

In pesticide detection large numbers of sensor assays have been reported in the literature [14-18]. Among them molecular imprinting-based biomimetic sensors have advantage of possessing high stability, sensitivity and specificity that is comparable to polyclonal antibodies. Polymerization is an easy and effective way to prepare 
synthetic materials able to mimic the molecular recognition in living system $[9,19,20]$. In this technique, the target molecule forms a complex with functional monomers and is then incorporated by polymerization into a highly cross-linked macro porous polymer matrix. Extraction of the print molecule leaves results in a negative pattern in the matrix. Sites with specific shape and functional groups complementary to the original print molecule are responsible for the special recognition capacity. Synthesized stable polymeric membranes imprinted with templates interact with target through covalent, ionic, or hydrogen bonds and utilized in sensor development. The molecularly imprinted polymer (MIP) has been widely used in sensor components [21-28].

In the present work an electrochemical sensor has been developed using isoproturon imprinted polymeric membranes and the same has been used to detect different concentration of isoproturon conductimetrically. Isoproturon was used as template and the composite non-imprinted polymeric membrane (UM) was synthesized from EGDMA for cross-linking and MAA as functional monomer. Methacrylic acid (MAA) was chosen for polymer formation as it is capable of strong electrostatic interactions with Isoproturon. Removal of the template from the UM was confirmed by UV spectrophotometer, FTIR and SEM study respectively. The molecularly imprinted membrane (MIP) was also used for the sensing of other structurally related compound monouron and diuron to check the cross selectivity of molecularly imprinted membrane.

\section{Materials and Methods}

\subsection{Reagents}

Isoproturon (3-p-cumenyl-1, 1-di-methyl urea) was purchased from Rallis India Ltd., Bombay. Methacrylic acid (MAA) was obtained from Serva (India). Ethylene glycol dimethacrylate (EGDMA) and N, N-dimethyl-p-toluidine (DMPT) were procured from Sigma Aldrich (India). Ethanol, toluene and benzyl alcohol were purchased from B.D.H., India. All chemicals and solvents were applied without further purification except isoproturon which was recrystallised three times with alcohol. Benzoyl peroxide was procured from B.D.H., India and was recrystallized three times with chloroform (Across, India). Isoproturon was procured from Modren Insecticides Ltd., Ludhiana, India. Monouran and diuron were supplied by AccuStandard, Inc. (New Haven, CT). Argon was purchased from Ultra Pure Gases (I) Pvt. Ltd., India. Analytical reagent grade chemicals were used along with deionized water to prepare solutions.

\subsection{Membrane Preparation}

For preparation of highly specific imprinted polymers, the formation of stable complexes between the templates and their functional monomers in the reaction mixture is crucial and must be preserved in the resulting polymers. Covalent and noncovalent interactions fix the template molecules within the selective cavities [29,30]. After the removal of template from composite molecularly imprinted polymer, uniform binding cavities are left in the membrane.

For MIP formation monomer methacrylic acid (MAA) was mixed with solvent and isoproturon as template $(0.6$ and $3.6 \%(\mathrm{w} / \mathrm{w}))$ and afterwards $2 \%(\mathrm{w} / \mathrm{w})$ benzoyl peroxide was added. The mixture was degassed by argon for 10 minutes followed by addition of 0.2 gram DMPT. Circular Whatman filter paper no. 5 was soaked in the polymerization mixture and hung upright in a cylindrical glass container filled with argon. The free radical polymerization was initiated by benzoyl peroxide which undergoes hemolytic fission forming a benzyloxy radical; polymerization was conducted overnight at room temperature. The non-imprinted control membrane was prepared analogously without isoproturon. The composite membranes were washed three times with ethanol to remove the printed isoproturon molecules and immersed in pure solvent before use.

\subsection{UV-Visible Spectrophotometer Study}

Isoproturon dissolved in ethanol during the soaking of prepared unimprinted membrane (UM) was measured using UV-Visible spectrophotometer (ECIL, India) at a wavelength of $240 \mathrm{~nm}$. The filter paper soaked in the polymer mixture was placed in absolute ethanol at $25^{\circ} \mathrm{C}$ $\pm 0.1^{\circ} \mathrm{C}$. The solvent was replaced every hour until imprinted and non-imprinted membranes had the same value of absorbance. Absolute ethanol was used as blank.

\subsection{FTIR of MIP Membrane with and without Isoproturon}

The absorption spectra of membranes prepared with and without isoproturon template were taken with FTIR (Nicollet). The absorption bands of the isoproturon imprinted membrane were recorded after five consecutive dippings of membrane in ethanol until the complete removal of template was confirmed by UV-Visible spectrophotometer.

\subsection{Scanning Electron Microscopy (SEM)}

The prepared membranes were stored in $\mathrm{NaN}_{3}$ until SEM study (Model JEOL JSM 840 SEM). MIP and non-imprinted membranes were analyzed at magnification 500 with resolution 20 to record the morphology of membrane surface.

\subsection{Contact Angle Measurement}

The surface energy of membranes with template and 
molecularly imprinted membranes, obtained after removal of isoproturon, was determined indirectly by using contact angle measurements to compare effects produced in the unimprinted and printed membranes with isoproturon. Contact angles of water and methanol on the surface of the prepared non-imprinted and imprinted membranes were measured at room temperature using the contact angle meter Horizontal Beam Comparator (SCHERR ST TUMICO). Drops of about $2 \mu \mathrm{l}$ were deposited on the membrane surface employing a tight syringe that was adjusted $0.5 \mathrm{~mm}$ above the membrane surface. The volume of the drop was slowly increased and decreased by adding the liquid to and subsequently withdrawing it from the surface using the syringe. Direct measurements of the advancing contact angle reflecting the hydrophobic character of the surface, and the receding contact angle $(\theta r)$, which determines the hydrophilic character of the surface, were performed at both left and right sides of each drop. In order to obtain reproducible results, no vibration and distortion of the drop during volume changes was allowed. Five readings were performed for each sample; the average value and standard deviation were calculated and reported in this study. As the surface energy cannot be measured directly, different indirect methods have been proposed in the literature [26-28]. In this study, the harmonic-mean [26] and the geometric-mean $[27,28]$ approximations were employed to get the dispersive and the non-dispersive contributions to the total surface energy.

\subsubsection{Geometric-Mean Equation}

This approximation was first proposed by Girifalco and Good and later modified by Owens and Wendt [27]. According to Owens and Wendt, the surface energy of a solid can be determined using the following equation applied to two liquids:

$$
(1+\cos \theta) \gamma_{l}=2\left(\gamma_{s}^{d} \gamma_{l}^{d}\right)^{1 / 2}+2\left(\gamma_{s}^{n d} \gamma_{l}^{n d}\right)^{1 / 2}
$$

where the terms $\gamma_{s}$ and $\gamma_{l}$ are the surface free energies of the solid and pure liquid respectively. The superscripts " $d$ " and " $n d$ " correspond to dispersive and non-dispersive contributions to the total surface energy, respectively. The contact angle, $\theta$, obtained by the following equation was used in Equation (1):

$$
\theta=\cos ^{-1}\left(\frac{\cos \theta_{a}+\cos \theta_{r}}{2}\right)
$$

where $\theta_{a}$ and $\theta_{r}$ are the advancing and receding contact angle, respectively. By measuring the contact angles of two liquids on a membrane surface, two simultaneous equations will be obtained for Equation (1), which can be solved for $\gamma_{s}^{d}$ and $\gamma_{s}^{n d}$. Consequently, by assuming the linear additivity of the intermolecular forces (i.e. dispersive and non-dispersive forces), the sum of the two components, $\gamma_{s}^{d}$ and $\gamma_{s}^{\text {nd }}$, should provide an estimate value of the total surface free energy. Owens and Wendt [27] employed water and methylene iodide as test liquids. Other liquid pairs have also been proposed in the literature [26-28].

\subsubsection{Harmonic-Mean Equation}

Alternatively, we proposed the use of the harmonic-mean expression in Young's equation for two liquids [26]. The following relationship was derived and employed to evaluate the total surface energy:

$$
(1+\cos \theta) \gamma_{l}=4\left(\frac{\gamma_{s}^{d} \gamma_{l}^{d}}{\gamma_{s}^{d}+\gamma_{l}^{d}}+\frac{\gamma_{s}^{n d} \gamma_{l}^{n d}}{\gamma_{s}^{n d}+\gamma_{l}^{n d}}\right)
$$

\subsection{Fabrication of Sensor Using MIP Membrane}

The membrane was inserted in a U-shaped glass cell implemented in a circuit setup (Figure 1) for the sensing of

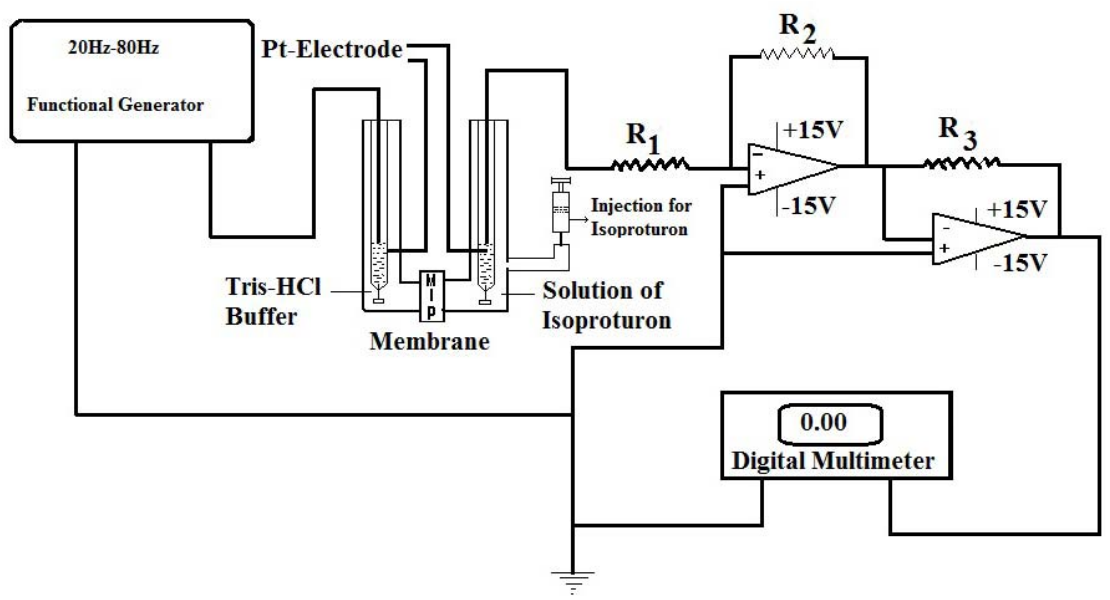

Figure 1. Electronic circuit of the electrochemical sensor used for the detection of isoproturon in absence and presence of structurally related Diuron and Monouron (own electronic circuit). 
isoproturon by a conductimetric method. One arm of the glass cell was filled with Tris- $\mathrm{HCl}(\mathrm{pH} \mathrm{8.0)}$ buffer, while the other one contained isoproturon prepared in Tris- $\mathrm{HCl}$. Different concentrations $(0.000001 \mathrm{M}, 0.00001 \mathrm{M}$, $0.0001 \mathrm{M}, 0.001 \mathrm{M})$ were applied subsequently. Two platinum electrodes were inserted in both arms of glass tube. Membrane electrical conductivity measurement was performed by applying a small-amplitude alternating voltage $(20 \mathrm{mV})$ with a varying frequency from $20 \mathrm{~Hz}$ to $80 \mathrm{kHz}$ generated by a low-frequency wave form generator. The output signal was transferred to an amplifier and read by a digital multimeter attached after the converter. The measured change in the membrane electrical conductivity as a function of isoproturon concentration was allowed to evaluate the recognition properties of the prepared membrane. The values of resistance have been calculated by Ohm's law. The value of current was directly read by a digital multimeter and the value of potential remains constant $(20 \mathrm{mV})$ for all the measurements. Three experiments were completed for each concentration of isoproturon out of $0.000001 \mathrm{M}, 0.00001 \mathrm{M}$, $0.0001 \mathrm{M}$ and $0.001 \mathrm{M}$ and the average values of membrane resistance have been quoted. The developed sensor system was tested for its cross selectivity and sensitivity with a mixture of equimolar concentration of Isoproturon, Monouron and Diuron ranging in the range of $100-900$ microgram per liter.

\section{Results and Discussions}

\subsection{Characterization of Membrane}

\subsubsection{UV-Visible Spectrophotometric Study}

The result obtained has been shown in Figure 2 which confirms the complete removal of template molecules from UM membrane after 12 hours and leads to the formation of MIP membrane.

\subsubsection{FTIR Study}

FTIR absorption spectra of the composite membrane prepared with isoproturon template show significant absorption bands on $3334,1717,1635,1453,1314,1295$, 1155, 1108, 1027, 813, 697 and 593 (nm) (Figure 3). In contrast absorption bands at 1717, 1295 and 813 are absent in composite membranes without template which allows confirming the removal of isoproturon (Figure 4). The absorption band at 1717, 1635 and 813 indicates the presence of the anilide bond, amide bond and aromatic system of isoproturon.

\subsubsection{Scanning Electron Microscopy}

The micrograph given in Figure 5 shows morphology of the isoproturon imprinted MIP membrane. The surface morphology clearly indicates the porous structure even after template removal. However it has to be emphasized here that these structures have nothing to do with molecular cavities generated by molecular imprinting but the possible binding of template molecules occurs on the visible porous surface.

\subsubsection{Calculation of Surface Energy from Contact Angles}

The measurement of contact angles revealed that advancing and receding contact angles were greater at the MIP membrane. Table 1 shows the values of the surface energies and the degree of surface polarity calculated as

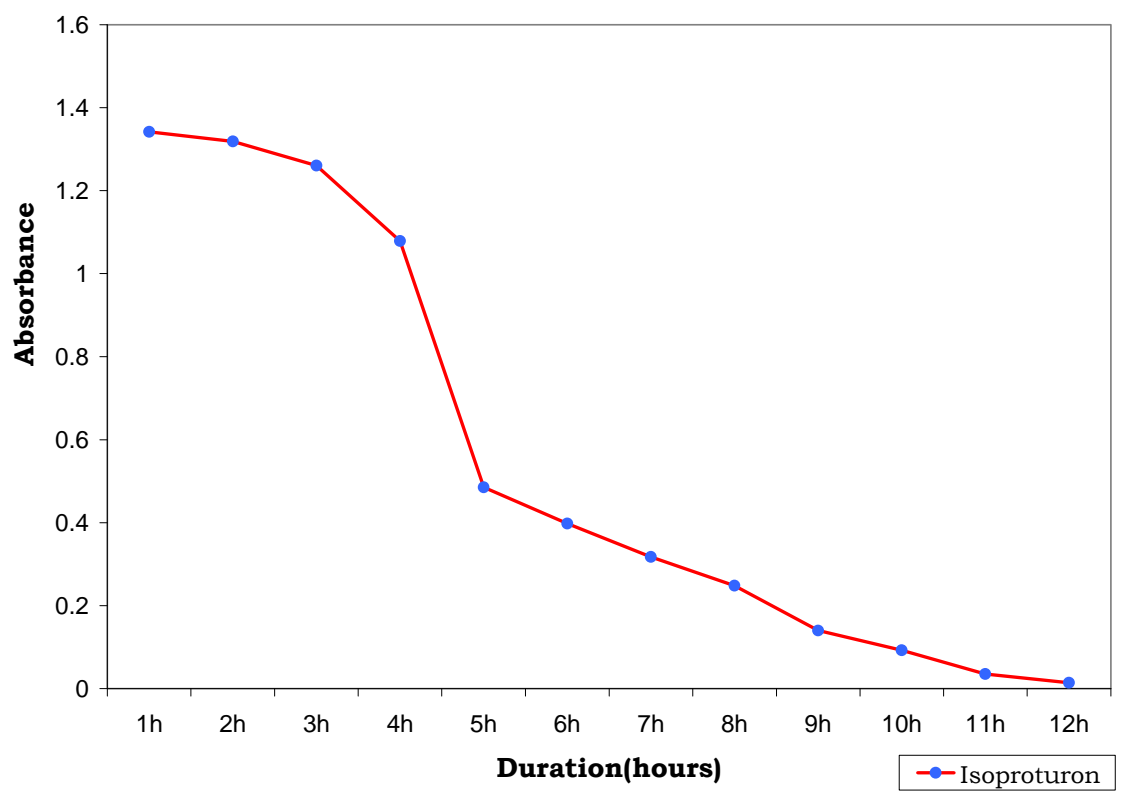

Figure 2. Time series of isoproturon removal from MPP measured at $240 \mathrm{~nm}$ in UV-visible spectrophotometer. 


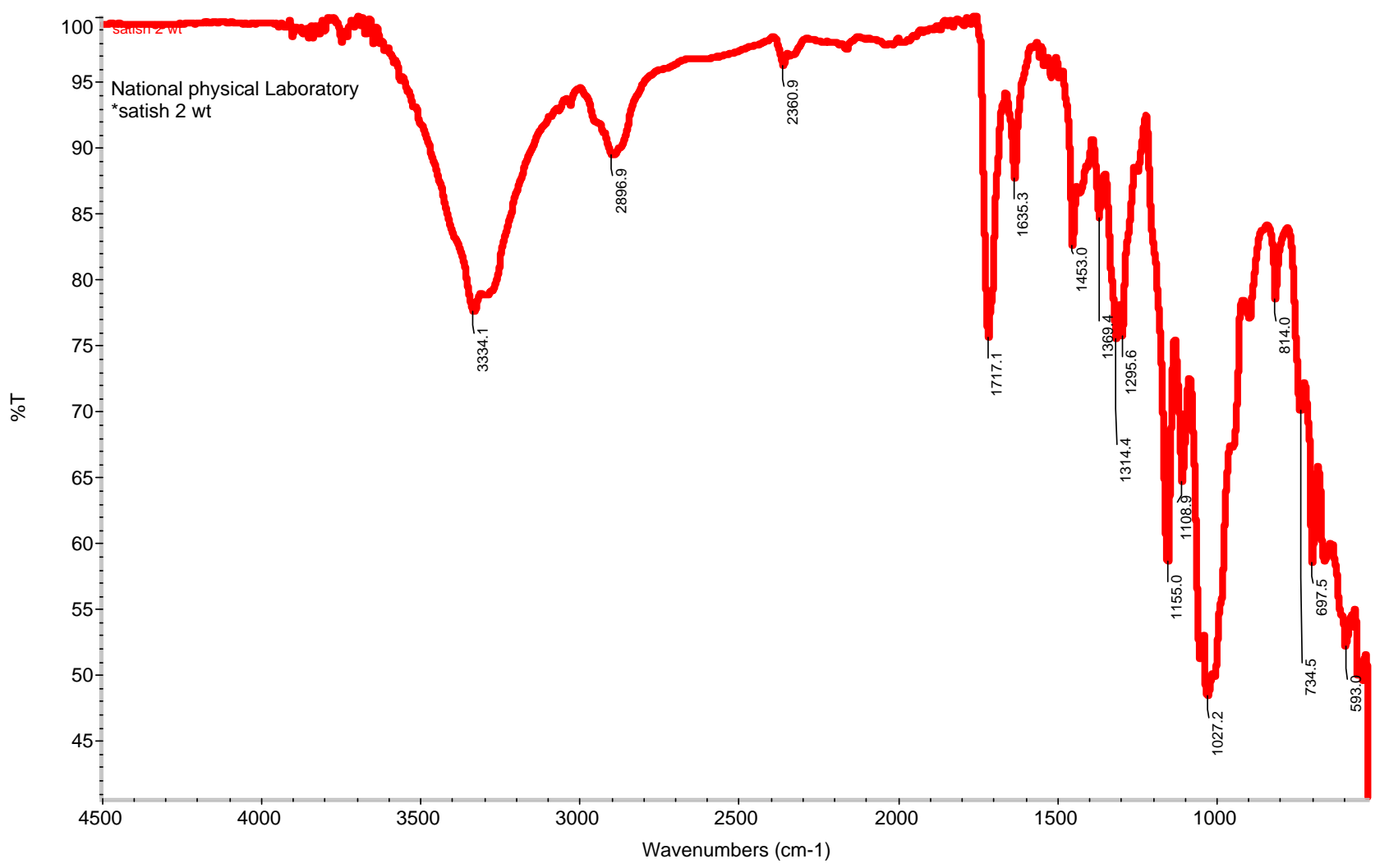

Figure 3. FTIR spectra of polymer composite membrane with isoproturon template.

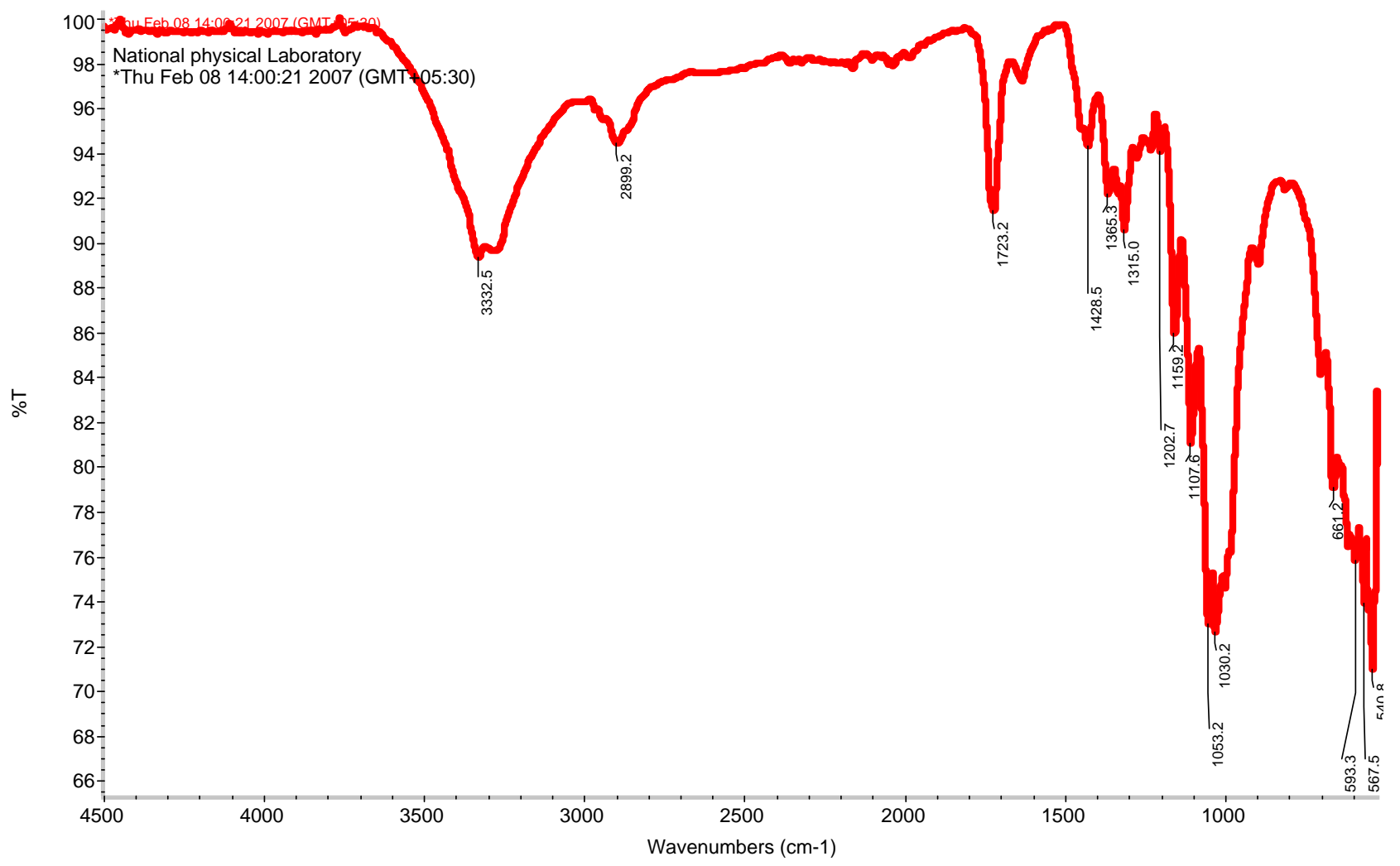

Figure 4. FTIR spectra of the isoproturon imprinted MIP membrane. 


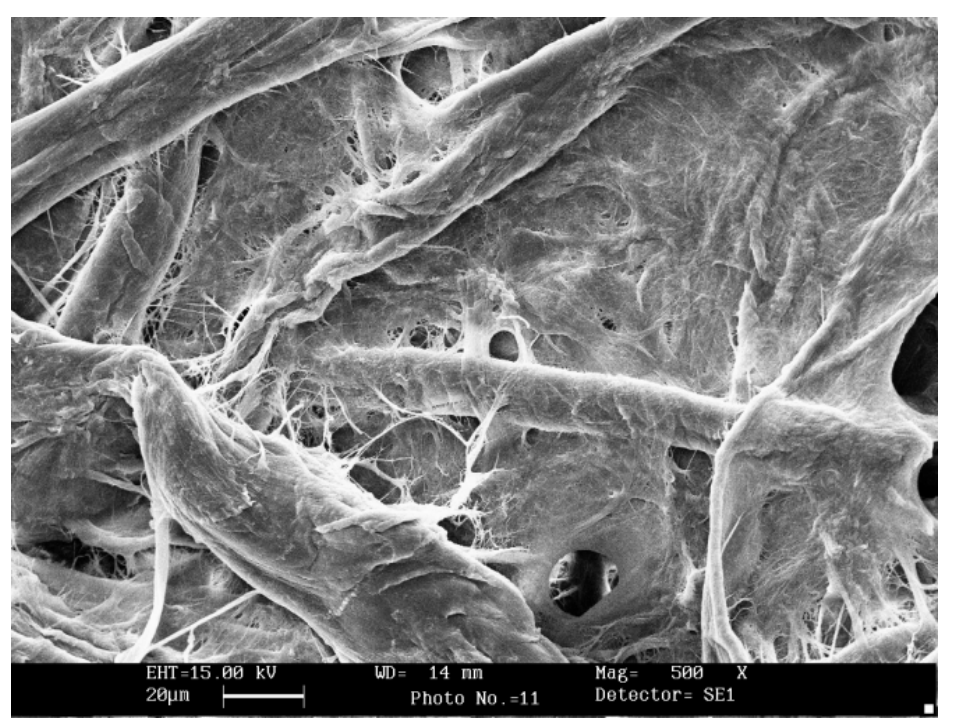

Figure 5. Scanning electron microscopy (SEM) of isoproturon imprinted membrane. Micrograph is taken at magnification $500 \mathrm{X}$ and resolution $20 \mu \mathrm{m}$ at SEI detector.

Table 1. The values of contact angles and surface energies of the unimprinted (UM) and molecularly imprinted polymer (MIP) membrane calculated by geometric-mean and harmonic mean approximation methods.

\begin{tabular}{ccccccccccc}
\hline Membrane & \multicolumn{2}{c}{ Water } & \multicolumn{2}{c}{ Methanol } & \multicolumn{3}{c}{$\begin{array}{c}\text { Geometric mean } \\
\text { Approximation }\left(\mathrm{mJ} / \mathrm{m}^{2}\right)\end{array}$} & \multicolumn{2}{c}{$\begin{array}{c}\text { Geometric mean } \\
\text { Approximation }\left(\mathrm{mJ} / \mathrm{m}^{2}\right)\end{array}$} \\
\hline & $\theta_{a}$ & $\theta_{r}$ & $\theta_{a}$ & $\theta_{r}$ & $\gamma_{s}^{n d}$ & $\gamma_{s}^{d}$ & $\gamma_{s}^{\text {nd }} / \gamma_{s}^{d} \%$ & $\gamma_{s}^{\text {nd }}$ & $\gamma_{s}^{d}$ & $\gamma_{s}^{\text {nd }} / \gamma_{s}^{d} \%$ \\
UM & 86.2 & 68.8 & 51.3 & 37.8 & 6.6 & 30.5 & 17.8 & 14.6 & 21.2 & 40.8 \\
MIP & 98.3 & 91.8 & 69.2 & 58.5 & 1.0 & 31.6 & 3.1 & 14.7 & 21.9 & 22.6 \\
\hline
\end{tabular}

the ratio of the non-dispersive surface energy to the total surface energy $\left(\gamma_{s}^{\text {nd }} / \gamma_{s}\right)$. Generally, the variation of $\gamma_{s}^{n d}$ values calculated using the geometric-mean equation agrees with those obtained from the harmonic-mean equation, but there are important quantitative differences. The values of $\gamma_{s}^{d}$ calculated with the geometric-mean equation are higher compared to the harmonic-mean approximation while the $\gamma_{s}^{\text {nd }}$ values obtained from the geometric-mean approximation are lower. The degree of surface polarity obtained is very small when the geometric-mean method is employed. The unimprinted (UM) membranes had a relatively high (about $15 \%$ ) non-dispersive, $\gamma_{s}^{\text {nd }}$ component in comparison with MIP membranes, while the dispersive component, $\gamma_{s}^{d}$, for both the UM and MIP membranes were almost equal. This was shown by the geometric-mean and the harmonic-mean approximations.

From our results we can conclude that the MIP membrane surface is more hydrophobic due to the presence of imprinted binding sites. Moreover, an increased receding contact angle indicates that hydrophobic locks remain at the membrane surface when the surrounding medium changes between air and liquid because the imprinted site roughness always exposed both liquid and air. Consistently it was observed that the stability of the hydropho- bic character was enhanced by increasing the amount of isoproturon.

\subsection{Electrochemical Sensing}

The results of electrochemical sensor obtained for the different concentrations of isoproturon are shown in Figure 6 which clearly show that the membrane resistance increases as the frequency increases from $20 \mathrm{~Hz}$ to $80 \mathrm{kHz}$. The observation and calculation made for isoproturon showed that as the frequency increases from 20 $\mathrm{Hz}$ to $80 \mathrm{kHz}$ the resistance also increases. As the concentration of solution increases the values of resistances also increase and it is maximum for solution having concentration of the order of $10^{-3} \mathrm{M}$. The maximum value of resistance ranged from $30-40$ to $60 \mathrm{kHz}$ for all the solution of isoproturon which indicates that the sensitivity of imprinted membrane is very high at this frequency range. The increase in electro résistance with concentration of template solution tends to saturate at lower concentration, probably due to filling the selective cavities in the polymeric domains by adsorption of the templates molecules. In the sensor technology, several factors are of main interest: sensitivity, selectivity, response reproducibility, and stability. As far as the sensitivity of the imprinted 


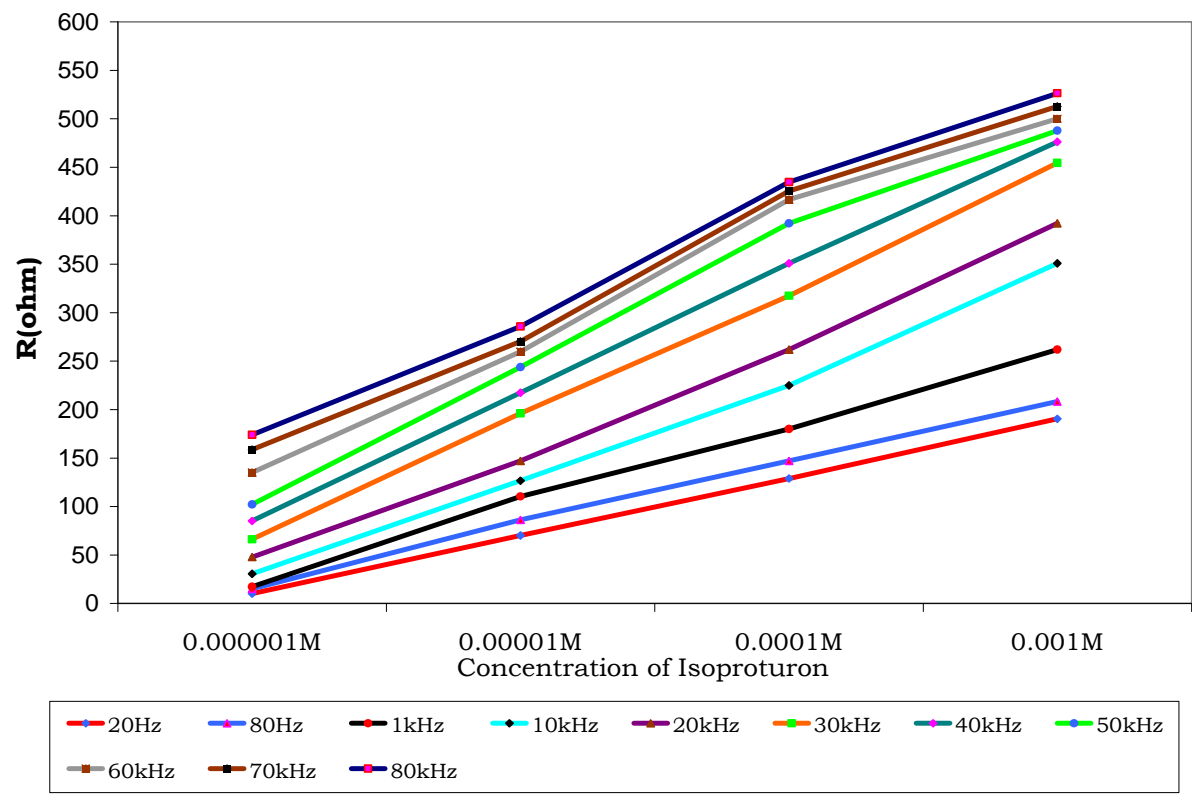

Figure 6. Influence of the current frequency on the sensor response of isoproturon membranes.

polymers is concerned, it is shown that the analytes of micro molar concentration can be used for detection.

From the above graph it is clear that the sensitivity of imprinted membrane is higher for high concentration and at high concentration the response time is very low. It was demonstrated that the sensitivity of the prepared membrane was of considerably high order because the least concentration of solution of isoproturon was the order of $10^{-6} \mathrm{M}$. It is clearly observed from Figure 6 that the concentration of herbicide increases the response in terms of membrane resistance shows greater increase in values (ranging from few ohms to $500 \mathrm{ohms}$ ). From this curve the concentration of isoproturon can be calculated in any given solution by measuring the output current and then calculating the value of resistance. By plotting the value of resistance in calibrated curve the concentration of isoproturon can be measured in any sample. The sensitivity and specificity can be increased by increasing the number of binding sites at MIP membrane's surface. In order to investigate the selectivity of the isoproturon imprinted membrane, substances with a molecular structure similar to isoproturon have been introduced in the electrochemical cell: monouron and diuron. Although the imprinted membrane for these structurally related compounds was not prepared but their sensing was tested with isoproturon imprinted membranes. Both of these substances show changes in the membrane resistance and the membrane frequency. The membrane resistance increases as the frequency increases from $20 \mathrm{~Hz}$ to $80 \mathrm{kHz}$. Both of these structurally related substances show changes in the membrane electro resistances less than $50 \mathrm{Ohm}$ as shown in Figure 7, confirming our results about high selectivity of the sensor responses for molecularly imprinted polymer.

\section{Discussions}

The conditions of membrane preparation and removing templates and unreacted monomers determine to a large extent the sensor reproducibility. For regeneration of the imprinted polymers after the measurements, the membranes were washed in organic solvents such as absolute alcohol. Due to membrane thickness, the washing procedure required more than $10 \mathrm{~h}$ for obtaining a full recovery of the polymers.

The stability of membrane has been investigated by repeating measurement of the sensor responses for isoproturon during 2 months. It has been found that template membrane can be stored at room temperature in buffer solution for 2 months without showing any loss in sensitivity. The selectivity of the imprinted polymers seems to be governed by number and nature of interaction between substrate and polymer stationary phases, as well as by the shape of the print molecule and cavities in the polymers. The nature of observed sensor responses might be explained by an assumption that interaction between the template molecule with the polymeric domain results in a conformational reorganization of the polymeric structure, affecting the diffusion of co- and counter ions.

For preparation of highly specific imprinted polymers, the formation of stable complexes between the templates and their functional monomers in the reaction mixture is crucial and must be preserved in the resulting polymers. Covalent and noncovalent interactions fix the template 


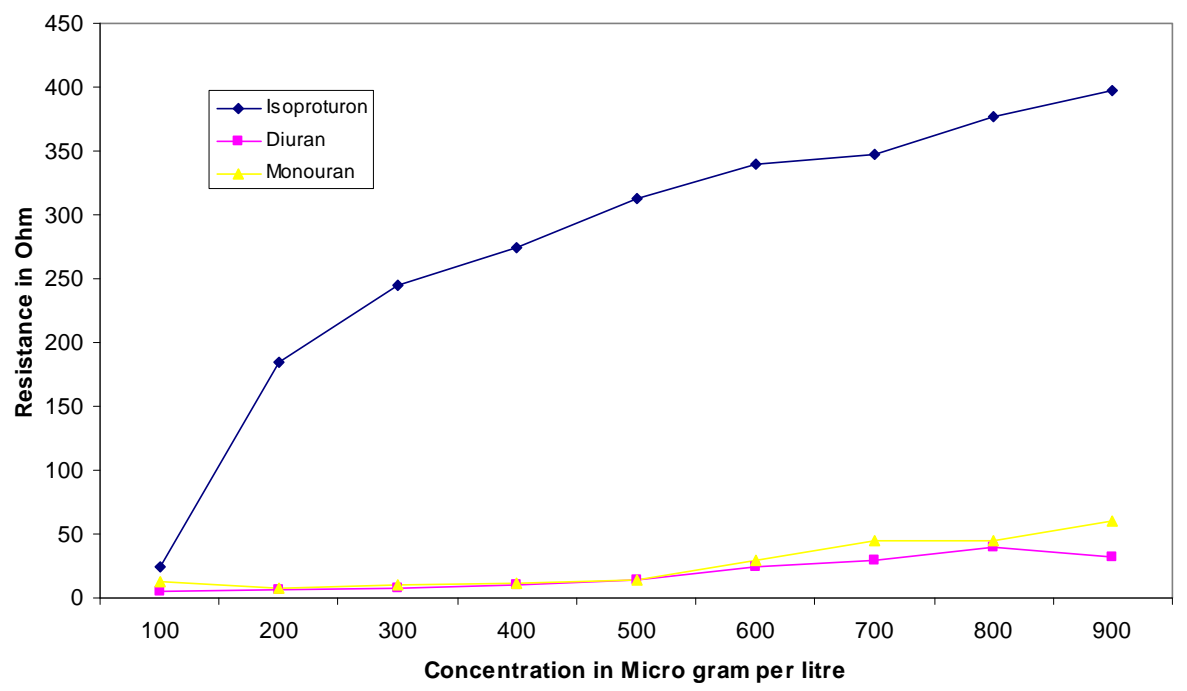

Figure 7. Selective sensitivity of isoproturon with structurally related diuran and monouran compounds.

molecules within the selective cavities [29,30]. During the course of polymerization where covalent interactions are established, there is a general stoichiometric relation between the template and the imprinted binding site (29). After the removal of template from composite molecularly imprinted polymer, uniform binding cavities are left in the membrane. In non covalent interaction, the association constant between the polymer and the template is found to be relatively low, so excess of functional monomer is required to saturate the recognition sites, and the removal of the template leaves a heterogeneous population of binding sites. Therefore, polymers prepared by covalent and by noncovalent binding behave quite differently with respect to electro conductivity.

In present experiment imprinted polymer was prepared by covalent interaction and the sensing of isoproturon was carried out by electro conductivity measurement and the molecular recognition ability of the imprinted polymers was studied electrochemically.

\section{Conclusion}

In summary imprinting of isoproturon was carried out to fabricate MIP membrane used in the development of electrochemical sensor of its detection. This MIP membrane enabled the detection of the isoproturon template in solution in the range of $10^{-3}-10^{-6} \mathrm{M}$ and this sensitivity can even be enhanced by changing the characteristics of the prepared membranes such as contact angle and thickness etc. The high specificity and stability exhibited by the membrane were confirmed by its characterization by UV-VIS, FTIR, SEM and contact angle measurement study which can also make such membrane a promising alternative to enzymes, antibodies, and natural receptors in sensor technology. This study has a large significance in health care, environmental pollution monitoring, phar- maceutical and fermentation industries.

\section{Acknowledgements}

Authors acknowledge with thanks the financial grant provided by Uttarakhand State Council for Science and Technology, Dehradun, Uttarakhand, India.

\section{REFERENCES}

[1] A. A. Boyd-Boland, S. Magdic and J. B. Pawliszyn, "Simultaneous Determination of 60 Pesticides in Water Using Solid-Phase Microextraction and Gas ChromatographyMass Spectrometry," Analyst, Vol. 121, No. 7, 1996, pp. 929-937. doi:10.1039/an9962100929

[2] R. Eisert and K. Levsen, "Determination of Pesticides in Aqueous Samples by Solid-Phase Microextraction In-Line Coupled to Gas Chromatography-Mass Spectrometry," Journal of the American Society for Mass Spectrometry, Vol. 6, No. 11, 1995, pp. 1119-1130.

[3] G. R. Pieper, "Residue Analysis of Carbaryl on Forest Foliage and in Stream Water Using HPLC," Bulletin of Environmental Contamination and Toxicology, Vol. 22, No. 1, 1979, pp. 167-171. doi:10.1007/BF02026924

[4] Z. Hussain and S. Siddique, "Determination of Pesticides in Fruits and Vegetables Using Acetonitrile Extraction and GC/MS Technique," Journal of Scientific Research, Vol. XXXX, No. 2, 2010, pp. 19-29.

[5] S. Yao, A. Meyer and G. Henze, "Comparison of Amperometric and UV-Spectrophotometric Monitoring in the HPLC Analysis of Pesticides," Fresenius' Journal of Analytical Chemistry, Vol. 339, No. 4, 1991, pp. 207-211. doi:10.1007/BF00325738

[6] C. D. Watts and B. Hegarty, "Use of Immunoassays for the Analysis of Pesticides and Some Other Organics in Water Samples," Pure and Applied Chemistry, Vol. 67, No. 8-9, 1995, 1533. doi:10.1351/pac199567081533

[7] C. Mouvet, S. Broussard, R. Jeannot, C. Maciag, R. 
Abuknesha and G. Ismail, "Validation of Commercially Available ELISA Microtiter Plates for Triazines in Water Samples," Analytica Chimica Acta, Vol. 311, No. 3, 1995, pp. 331-339. doi:10.1016/0003-2670(94)00638-3

[8] M. Franek, V. Kolar and S. A. Eremin, "Enzyme Immunoassays for $s$-Triazine Herbicides and Their Application in Environmental and Food Analysis," Analytica Chimica Acta, Vol. 311, No. 3, 1995, pp. 349-356. doi:10.1016/0003-2670(95)00158-V

[9] G. Wulff, "Molecular Imprinting in Cross-Linked Materials with the Aid of Molecular Templates-A Way towards Artificial Antibodies," Angewandte Chemie, International Edition in English, Vol. 34, No. 17, 1995, pp. 1812-1832. doi:10.1002/anie.199518121

[10] K. J. Shea, "Molecular Imprinting of Synthetic Network Polymers: The de Novo Synthesis of Macromolecular Binding and Catalytic Sites," Trends in Polymer Science, Vol. 2, No. 5, 1994, 166-173.

[11] D. Kriz and K. Mosbach, "Competitive Amperometric Morphine Sensor Based on an Agarose Immobilised Molecularly Imprinted Polymer," Analytica Chimica Acta, Vol. 300, No. 1-3, 1995, pp. 71-75. doi:10.1016/0003-2670(94)00368-V

[12] S. A. Piletsky, E. V. Piletskaya, A. V. Elgersma, K. Yano, I. Karube, P. Yu. Parhometz and A. V. El'skaya, "Atrazine Sensing by Molecularly Imprinted Membranes," Biosensors and Bioelectronics, Vol. 10, No. 9-10, 1995, pp. 959-964. doi:10.1016/0956-5663(95)99233-B

[13] S. A. Piletsky, T. L. Panasyuk, E. V. Piletskaya, A. V. El'skaya, R. Levi, I. Karube and G. Wulff, "Imprinted Membranes for Sensor Technology: Opposite Behavior of Covalently and Noncovalently Imprinted Membranes," Macromolecules, Vol. 31, No. 7, 1998, pp. 2137-2140. doi:10.1021/ma970818d

[14] A. W. Flounders, A. K. Singh, J. V. Volponi, S. C. Carichner, K. Wally, A. S. Simonian, J. R. Wild and J. S. Schoeniger, "Development of Sensors for Direct Detection of Organophosphates. Part-II: Sol-Gel Modified Field Effect Transistor with Immobilised Organophosphate Hydrolase," Biosensors and Bioelectronics, Vol. 14, No. 8-9, 1999, pp. 715-722. doi:10.1016/S0956-5663(99)00045-7

[15] E. Mallat, D. Barcelo, C. Barzen, G. Gauglitz and R. Abuknesha, "Immunosensors for Pesticide Determination in Natural Waters," TrAC Trends in Analytical Chemistry, Vol. 20, No. 3, 2001, pp. 124-132. doi:10.1016/S0165-9936(00)00082-0

[16] O. A. Sadik and J. M. Van Emon, "Application of Electrochemical Immunosensors to Environmental Monitoring," Biosensors \& Bioelectronics, Vol. 11, No. 8, 1996, pp. 1-11.

[17] S. M. Reddy, J. P. Jones, T. J. Lewis and P. M. Vadgama, "Development of an Oxidase-Based Glucose Sensor Using Thickness-Shear-Mode Quartz Crystals," Analytica Chimica Acta, Vol. 363, No. 2-3, 1998, pp. 203-213. doi:10.1016/S0003-2670(98)00131-7

[18] N. G. Karousos, et al., "Quartz Crystal Microbalance Determination of Organophosphorus and Carbamate Pes- ticides," Analytica Chimica Acta, Vol. 469, No. 2, 2002, pp. 189-196. doi:10.1016/S0003-2670(02)00668-2

[19] A.-H. Wu and M.-J. Syu, "Synthesis of Bilirubin Imprinted Polymer Thin Film for the Continuous Detection of Bilirubin in an MIP/QCM/FIA System," Biosensors and Bioelectronics, Vol. 21, No. 12, 2006, pp. 2345-2353. doi:10.1016/j.bios.2006.01.017

[20] B. Sellergren, "Noncovalent Molecular Imprinting: Antibody-Like Molecular Recognition in Polymeric Network Materials," TrAC Trends in Analytical Chemistry, Vol. 16, No. 6, 1997, pp. 310-320. doi:10.1016/S0165-9936(97)00027-7

[21] K. P. Singh, S. Ahalawat, R. K. Prajapati, S. Kumar, P. Singh and S. K. Dhawan. "Electrochemical Sensing for the Detection of 2,4-Dichlorophenoxy Acetic Acid Using Molecularly Imprinted Polymer Membrane," Ionics, Vol. 16, No. 6, 2010, pp. 529-537. doi:10.1007/s11581-010-0419-0

[22] T. Takeuchi and J. Haginaka, "Separation and Sensing Based on Molecular Recognition Using Molecularly Imprinted Polymers," Journal of Chromatography B: Biomedical Sciences and Applications, Vol. 728, No. 1, 1999, pp. 1-20. doi:10.1016/S0378-4347(99)00057-2

[23] Q. Liu, Y. X. Zhou and Y. T. Liu, "Recent Progresses in Research on Molecular Imprinting Sensors," Chinese Journal of Analytical Chemistry, Vol. 27, No. 11, 1999, pp. 1341-1347.

[24] F. L. Dickert, P. Lieberzeit and M. Tortschanoff, "Molecular Imprints as Artificial Antibodies-A New Generation of Chemical Sensors," Sensors and Actuators B: Chemical, Vol. 65, No. 1-3, 2000, pp. 186-189. doi:10.1016/S0925-4005(99)00327-5

[25] S. Wu, "Polymer Interface and Adhesion," Marcel Dekker, New York, 1982.

[26] D. K. Owens and R. C. Wendt, "Estimation of the Surface Free Energy of Polymers," Journal of Applied Polymer Science, Vol. 13, No. 8, 1969, pp. 1741-1747. doi:10.1002/app.1969.070130815

[27] K. L. Mittal, "Contact Angle, Wettability and Adhesion," Vision Sports Publishing (VSP), Utrecht, 1993.

[28] B. Sellergren, "Molecular Imprinting by Noncovalent Interactions. Enantioselectivity and Binding Capacity of Polymers Prepared under Conditions Favouring the Formation of Template," Die Makromolekulare Chemie, Vol. 190, No. 11, 1989, pp. 2703-2711. doi:10.1002/macp.1989.021901104

[29] S. A. Piletsky, D. M. Fedoryak, I. D. Atamanenko, M. T. Bryk and V. P. Kukhar, "Nucleoside-Selective Polymers Based on Methacrylate Copolymers," Ukrainskii Khimicheskii Zhurnal, Vol. 59, No. 12, 1993, pp. 1316-1320. (in Russian)

[30] I. A. Nicholls, L. I. Andersson, K. Mosbach, "Recognition and Enantioselection of Drugs and Biochemicals Using Molecularly Imprinted Polymer Technology," Trends in Biotechnology, Vol. 13, No. 2, 1995, pp. 47-51. doi:10.1016/S0167-7799(00)88904-0 